\title{
Aspectos clínicos y etiológicos de la hemorragia digestiva alta y sus escalas de evaluación
}

\author{
Yuban Sebastian Cuartas-Agudelo* \\ Lina María Martínez-Sánchez ${ }^{* *}$
}

\begin{abstract}
*Estudiante VII semestre Medicina. Grupo de investigación de Medicina Interna, Línea de hematología, Escuela de Ciencias de la Salud. Universidad Pontificia Bolivariana. Medellín. Antioquia. Colombia.

**Magister. Docente titular, Universidad Pontificia Bolivariana. Grupo de investigación de Medicina Interna; Línea de hematología; Escuela de Ciencias de la Salud. Universidad Pontificia Bolivariana. Medellín. Antioquia. Colombia.

Correspondencia: Sr. Yuban Sebastian Cuartas Agudelo. Calle 78 B № 72A 109, Medellín. Antioquia. Colombia. Correo electrónico: yuban.cuartas@ upb.edu.co
\end{abstract}

Resumen

Introducción: La hemorragia digestiva alta es frecuente, con diversas etiologías, métodos de diagnóstico y tratamiento para el manejo de estos pacientes, además, cuenta con escalas de predicción clínica. Objetivo: describir aspectos clave relacionados con la etiología, diagnóstico, factores de riesgo y escalas de evaluación que se han usado en pacientes con esta entidad. Metodología de búsqueda: Fueron incluídos artículos publicados en idiomas español e inglés, la mayoría entre 2013 y 2019 que tengan contenido relacionado con el objetivo del presente manuscrito. Conclusiones: La hemorragia digestiva alta es la principal emergencia gastroenterológica, con diferentes etiologías y métodos de diagnóstico y tratamiento orientados a mejorar la supervivencia de estos pacientes, actualmente se recomiendan diferentes escalas de evaluación para predecir desenlaces de estos pacientes, pero los estudios han tenido diferencias en los resultados encontrados, lo que sucita una oportunidad de investigación para beneficio de los pacientes y la práctica clínica. MÉD.UIS.2020;33(3):9-20

Palabras clave: Tracto Gastrointestinal Superior. Hemorragia Gastrointestinal. Endoscopía. Enfermedades del Esófago. Gastropatías. Enfermedades Duodenales.

\section{Clinical and etiological aspects of upper gastrointestinal bleeding and its evaluation scales}

Abstract

Introduction: Upper gastrointestinal bleeding it's a frequent clinical situation, has different etiologies, diagnostic methods and treatments for the management of these patients, in addition, has clinical scales to predict outcomes in those patients. Objective: to describe key points related to the etiology, diagnosis, risk factors, and evaluation scales that have been used in patients with this entity. Methodology: Articles published in spanish and english were included, the majority between 2013 and 2019 that have content related to the objective of this manuscript. Conclusions: Upper gastrointestinal bleeding is the main gastroenterological emergency, with different etiologies and methods of diagnosis and treatment aimed at improving the survival of these patients; currently, different evaluation scales are recommended to predict outcomes in these patients, but studies have differences in the results between them, which creates an opportunity of clinical research for patients and clinical practice benefit. MÉD.UIS.2020;33(3):9-20.

Keywords: Upper gastrointestinal tract. Gastrointestinal hemorrhage. Endoscopy. Risk assessment.

¿Cómo citar este artículo?: Cuartas-Agudelo YS, Martínez-Sánchez LM. Aspectos clínicos y etiológicos de la hemorragia digestiva alta y sus escalas de evaluación. MÉD.UIS.2020;33(3):9-20. doi: 10.18273/revmed.v33n3-2020001 


\section{Introducción}

La hemorragia digestiva alta (HDA) o superior, es una situación clínica que se presenta cuando existe una estructura sangrante que tiene como lugar el esófago, estómago o la primera porción del duodeno, teniendo como límites anatómicos el esfínter esofágico superior y el ligamento de Treitz ${ }^{1-3}$. Dicho sangrado puede proceder de estructuras contiguas que vierten el contenido hemorrágico en el tubo gastrointestinal o ser directamente originado en el tracto digestivo $0^{1,4}$. La hemorragia digestiva se divide en superior e inferior, siendo la primera hasta cinco veces más frecuente que la segunda ${ }^{5}$. Actualmente esta patología es una causa importante de admisión hospitalaria y, a pesar de los avances médicos para su determinación y tratamiento, no se ha logrado mejorar la tasa de mortalidad (alrededor del 10\%) en los pacientes ${ }^{6}$.

La HDA ha sido catalogada como la principal emergencia gastroenterológica por su alta prevalencia, siendo en Estados Unidos de 50 a 172/100000 personas por año. La incidencia de esta condición clínica está reportada entre 34, 72 y 100/100 000 personas en España, Reino Unido y Malasia respectivamente ${ }^{7,8}$. La mortalidad de las personas que presentan la HDA es más alta en China donde oscila entre 4 y 14\%; en latinoamérica, específicamente en Perú y México la mortalidad es cercana al 9 y $4 \%$ respectivamente; en Colombia, por su parte, hay pocos estudios que documenten los aspectos epidemiológicos de esta entidad, sin embargo, se puede afirmar que la mortalidad ha permanecido entre el 5 y el $10 \%$ en los últimos 20 años; por otro lado, en un estudio realizado en Bogotá, capital del país, el $5.7 \%$ de las endoscopias realizadas fueron por hemorragia digestiva y en Barranquilla la HDA fue la indicación del $20 \%$ de las esofago-gastroscopias ${ }^{8-12}$.

La HDA se presenta mayoritariamente en personas de sexo masculino con una edad media de 60 años, y se estima que el $13,9 \%$ de pacientes que la presentan, resangran en los primeros siete días, pero dicha cifra asciende al 30\% según la bibliografía estadounidense y británica; además, el impacto económico que conlleva alcanza los 2000 millones de dólares anuales en Estados Unidos $8,13-18$.

La presentación clínica de la HDA difiere de la inferior en que generalmente no se encuentra contenido hemático en el recto, pero sí cursa con hematemesis y melenas; en ocasiones se presenta con signos sistémicos propios de una pérdida de sangre como lo son debilidad, mareo y síncope. Diferentes hallazgos como epistaxis, gingivorragia, sangrado tonsilar e incluso alimentos de color rojo y diversos medicamentos que contienen bismuto pueden semejar una falsa hemorragia digestiva alta ${ }^{19}$.

Los antiinflamatorios no esteroideos (AINES) son considerados un factor de riesgo importante para presentar sangrado digestivo alto al debilitar los mecanismos protectores de la mucosa gastrointestinal ${ }^{20}$. Un estudio en Turquía demostró que el $16,3 \%$ de pacientes con hemorragia digestiva superior estaban medicados con AINES ${ }^{21}$. Respecto al alcoholismo se determinó que el $23,7 \%$ de pacientes que resangraron presentaban este antecedente ${ }^{16}$. También han sido documentados otros factores de riesgo como el sexo masculino, cirrosis, inhibidores COX-2 selectivos e infección por Helicobacter pylori2 ${ }^{22}$.

El pronóstico de la HDA puede variar entre leve y mortal: pacientes con sangrado leve podrían recuperarse completamente sin tratamiento clínico específico, mientras que aquellos con sangrado severo pueden experimentar una complicación severa e incluso la muerte si no reciben el tratamiento para su condición ${ }^{2,9}$.

La identificación temprana de factores de riesgo y la predicción del pronóstico pueden inducir una intervención apropiada, reduciendo así la mortalidad, la morbilidad, la estancia hospitalaria y los costos. En este sentido, se han desarrollado varios sistemas de puntuación para predecir la gravedad en pacientes con $\mathrm{HDA}^{23}$.

Durante la última década, el manejo de la HDA y la comprensión del riesgo de estratificación siguen siendo un tema frecuente de investigación y publicación, puesto que el diagnóstico precoz, así como la estratificación precisa de pacientes con mayor riesgo de mortalidad y resangrado, pueden impactar en las decisiones clínicas y ayudar al momento de tomar decisiones finales pertinentes ${ }^{2}$. Existen múltiples sistemas de puntuación que estratifican a los pacientes en aquellos con alto y bajo riesgo, sin embargo, continúan las controversias relacionadas con la estratificación del riesgo, papel de la endoscopia, el tratamiento específico, la necesidad de cirugía adicional, la intervención endoscópica y la muerte en aquellos pacientes clasificados. En consecuencia, hay diferentes perspectivas entre 
los autores sobre cómo usar adecuadamente estas escalas en los pacientes ya que la mayoría de los estudios se han centrado en la mortalidad y estancias hospitalarias, dejando rezagados otros desenlaces².

Las escalas de evaluación para HDA han surgido desde el siglo pasado debido a la necesidad de diagnosticar y clasificar rápidamente a los pacientes para así establecer los desenlaces más y menos probables de acuerdo al resultado obtenido; en este artículo se plantean cuatro de ellas: Forrest, Rockall, Glasgow Blatchford y AIMS65, cada una evalúa diferentes variables tales como hallazgos de laboratorio, examen físico, hallazgos endoscópicos, comorbilidades y edad, las cuales se relacionan con el resangrado, mortalidad, necesidad de intervención, estancia hospitalaria y costo del tratamiento. En los últimos años se han llevado a cabo numerosos estudios con poblaciones que presentan HDA para evaluar la capacidad predictiva de dichas escalas; sin embargo, estas publicaciones difieren en los resultados obtenidos y evidencian la inexistencia de una escala de evaluación única con excelente desempeño, lo que presenta actualmente una limitante en la estratificación de los pacientes y abre nuevas posibilidades orientadas hacia el mejoramiento o la creación de nuevas escalas que destaquen entre las demás.

Este artículo tiene como objetivo exponer una descripción clara sobre aspectos clave relacionados con la etiología, diagnóstico, factores de riesgo y, particularmente, escalas de evaluación que se han usado en pacientes con HDA alrededor del mundo.

\section{Metodología de búsqueda}

La consulta se realizó entre los meses de junio y noviembre de 2019 en la que se seleccionaron artículos originales, reportes y series de casos, revisiones sistemáticas y narrativas en las bases de datos PubMed y ClinicalKey. Los términos de búsqueda en español fueron hemorragia digestiva superior, hemorragia gastrointestinal por separado y estas dos combinadas con el booleano [AND] con escalas de evaluación; las principales palabras de búsqueda en inglés fueron upper gastrointestinal bleeding, gastrointestinal hemorrhage y evaluation scales. Se excluyeron artículos que estaban disponibles en un idioma diferente al inglés o español, se hizo lo propio con los artículos que según criterio de los autores basándose en la revisión del título y del resumen de los artículos, no contasen con la información necesaria para dar respuesta al objetivo, sea porque no ahondaban en aspectos epidemiológicos, etiológicos, diagnósticos o terapéuticos relevantes para la revisión de tema o porque no poseían información en cuanto a las escalas de evaluación; también se excluyeron publicaciones por duplicación de la información y por falta de acceso al texto completo según las licencias otorgadas por la Universidad Pontificia Bolivariana. Se priorizaron artículos publicados entre 2013 y 2019, se incluyeron los artículos que en el título o resumen evidenciaran un abordaje integral de la hemorragia digestiva alta y aquellas publicaciones concernientes a las escalas de evaluación. Los autores añadieron artículos que consideraron pertinentes para enriquecer el proceso de introducción y discusión de la presente revisión de tema y algunos más antiguos para contextualizar el desarrollo de las diferentes escalas de evaluación. (Ver figura 1)

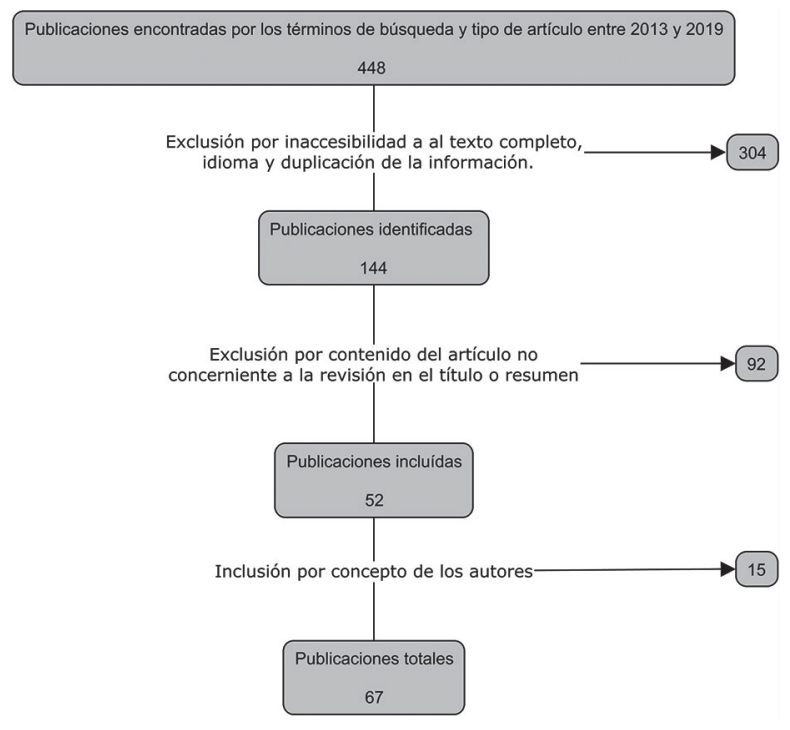

Figura 1. Búsqueda, selección y exclusión de artículos.

Fuente: autores.

\section{Etiologías}

Los autores dividen la HDA en dos grupos: no varicosa y varicosa, teniendo cada una presentación diferente ${ }^{24-27}$. (Ver Tabla 1)

La hemorragia no varicosa engloba las úlceras esofágicas bajas, gástricas y duodenales, erosiones gastroesofágicas, reflujo gastroesofágico, gastropatía hipertensiva portal y tumor gástrico, por su parte, el sangrado de origen varicoso se presenta principalmente mediante las várices esofágicas. El síndrome de Mallory Weiss también es 
considerada una causa importante ${ }^{28}$. Las patologías más prevalentes son la úlcera péptica (duodenal y gástrica) y las várices esofágicas ${ }^{8,16,27-29}$.

\section{Úlcera péptica}

Es una lesión del tracto digestivo generada por el ácido peptídico el cual ocasiona una rotura epitelial que alcanza la submucosa, generalmente localizada en el estómago o la porción proximal del duodeno ${ }^{4,30}$. Las dos principales causas de esta patología son la colonización por Helicobacter pylori y el uso de AINES: la bacteria posee varios mecanismos para dañar la mucosa: el primero es una inhibición de las células D que son productoras de somatostatina, lo que finalmente genera una hipersecreción de gastrina que produce una carga acida en el duodeno, el segundo es la disminución en la secreción de bicarbonato y el tercero es el bloqueo de la liberación de secretina en el duodeno, además induce la liberación de sustancias proinflamatorias que empeoran el cuadro ${ }^{4}$. Los AINES generan una disminución de las prostaglandinas por inhibición de la vía de la ciclooxigenasa 1, lo que lleva a una alteración de la capacidad que tienen las células para repararse debido a una disminución del flujo sanguíneo ${ }^{31}$. Esta patología puede llevar a una complicación grave: la úlcera péptica perforada, la cual confiere el riesgo de una exposición del peritoneo al contenido gástrico ${ }^{32}$.

Las ulceras también se encuentran asociadas a estrés fisiológico, hipersecreción ácida (gastrinoma) y malignidad. La ulceración inducida por estrés se observa en pacientes gravemente enfermos en unidad de cuidados intensivos, con ventilación mecánica a largo plazo y coagulopatía4.

La presentación clínica varía de acuerdo a la localización de la lesión, los pacientes con una úlcera ubicada en la porción gástrica presentan dolor abdominal postprandial, emesis, náuseas y pérdida de peso; mientras que las ubicadas en el duodeno generan síntomas como deseo de alimentarse constantemente y dolor abdominal nocturno el cual está asociado a la secreción gástrica ${ }^{33}$. En caso de una perforación, el paciente presenta dolor de ubicación epigástrica y rigidez abdominal debida a la afección peritoneal $^{32}$. La mortalidad de las úlceras pépticas se encuentra alrededor del $10 \%$, sin embargo, puede aumentar al 30\% cuando se perfora ${ }^{34}$.

\section{Várices esofágicas}

Son consideradas una complicación de la hipertensión portal. La ruptura de las várices suele ocurrir en aquellos pacientes que presentan una presión portal superior a los $12 \mathrm{mmHg}$, que se evidencia en aproximadamente el $50 \%$ de los pacientes diagnosticados con cirrosis; cabe aclarar que dicha ruptura también puede presentarse con valores de presión portal menores al planteado 4,35-37.

La formación de estas várices está relacionada con un aumento de la resistencia al flujo portal lo cual genera un incremento de presión que lleva a la apertura de canales vasculares embrionarios que se dilatan progresivamente y que además, ven su crecimiento favorecido por la expresión de factores de crecimiento derivados del endotelio vascular $(\mathrm{VEGF})^{38}$.

El aspecto clínico de estos pacientes es producto del aumento de presiones que tienen como consecuencia el sangrado de origen varicoso, el cual, cuando se presenta en conjunto con ascitis en pacientes con cirrosis, implica una mortalidad del $57 \%$ según una revisión sistemática con 118 estudios y 23797 pacientes ${ }^{38,39}$.

\section{Esofagitis}

Representa aproximadamente el 10\% de los casos de HDA, el reflujo gastroesofágico grave y el abuso de alcohol son los dos factores de riesgo más comunes para la esofagitis erosiva. Otras causas de esofagitis asociada con sangrado son la esofagitis infecciosa y la asociada a fármacos ${ }^{4}$.

La isquemia puede conducir a necrosis esofágica, aunque es poco frecuente. En pacientes con HDA secundaria a esofagitis, la hematemesis es más común que la melena, la presencia de odinofagia y disfagia dependerá de la cronicidad y gravedad de la condición subyacente 4 .

\section{Síndrome de Mallory Weiss}

Es una causa de HDA que en la mayoría de los casos es autolimitada ${ }^{40}$. Esta patología consta de laceraciones longitudinales que no perforan la unión gastroesofágica, origina del 3 al 15\% de los sangrados 
y su mecanismo es la generación de un aumento de presiones en la cavidad abdominal y gástrica que se refleja a nivel de la unión entre el estómago y el esófago ${ }^{41}$.

Los factores de riesgo documentados son el hipo, la tos, convulsiones y traumas torácicos; el consumo de alcohol está presente en un 30-60\% de los pacientes mientras que la hernia hiatal en un $40-80 \%$. Su causa también puede ser iatrogénica por procedimientos de estudios ecocardiográficos a nivel de la unión gastroesofágica o cardias $^{42}$.

La presentación clínica generalmente está precedida por vómito, náuseas o tos y los pacientes presentan de manera frecuente hematemesis, aunque también puede cursar con otras manifestaciones de menor incidencia como dolor epigástrico o de espalda, melena y hematoquecia. Debido a la hemorragia también puede comprometerse la hemodinamia lo que se verá reflejado mediante taquicardia e hipotensión ${ }^{43}$. La mortalidad del síndrome de Mallory Weiss en pacientes con HDA es del 5,3\% $\%^{42}$.

Tabla 1. Etiología de la HDA.

\begin{tabular}{|c|c|}
\hline Esófago & $\begin{array}{l}\text { Varices esofágicas } \\
\text { Esofagitis erosiva } \\
\text { Esofagitis infecciosa } \\
\text { Esofagitis por fármacos } \\
\text { Malignidad esofágica } \\
\text { Síndrome de Mallory Weiss } \\
\text { Isquemia }\end{array}$ \\
\hline Estómago & $\begin{array}{l}\text { Úlcera péptica } \\
\text { Varices gástricas } \\
\text { Malignidad gástrica } \\
\text { Gastropatía portal hipertensiva } \\
\text { Ectasia vascular antral } \\
\text { Lesión de Dieulafoy }\end{array}$ \\
\hline Intestino delgado & $\begin{array}{l}\text { Úlcera duodenal } \\
\text { Malignidad duodenal }\end{array}$ \\
\hline Miscelánea & $\begin{array}{l}\text { Hemofilia } \\
\text { Hemosuccus pancreaticus } \\
\text { Fístula aortoenterica } \\
\text { latrogénica }\end{array}$ \\
\hline
\end{tabular}

Fuente: Kamboj AK, Hoversten P, Leggett CL. Upper Gastrointestinal Bleeding: Etiologies and Management. Mayo Clin Proc. 2019;94(4):697-703.

\section{Diagnóstico e intervención}

Antes de cualquier diagnóstico, se presta un abordaje que asegure la estabilidad hemodinámica del paciente mediante administración de oxígeno, reposición del volumen intravascular y transfusiones sanguíneas que mantengan la hemoglobina entre 7 y 9 g/dl según la Guía de la Sociedad Europea de Endoscopia Gastrointestinal (ESGE); también recomiendan el inicio inmediato de inhibidores de bomba de protones vía intravenosa ${ }^{4,44,45}$.

Los pacientes son clasificados según aspectos clínicos como su estado hemodinámico, antecedentes patológicos, resultados de laboratorio recientes y edad, para esto se emplean algunas escalas como el índice de Rockall, la escala de Glasgow Blatchford y el AIM65 que determinan si el paciente debe ser ingresado al hospital, recibir transfusiones sanguíneas o tratamiento endoscópico ${ }^{46}$.

El diagnóstico se lleva a cabo determinando si el origen de la hemorragia intestinal es alto o bajo: hallazgos como hematemesis y melenas son indicadores más frecuentes en sangrado digestivo superior que inferior; en el lavado por sonda nasogástrica se encuentra contenido hemático o de aspecto similar al café, otro hallazgo sugestivo es la relación entre el nitrógeno uréico sanguíneo (BUN) y la creatinina mayor a $30^{47}$.

Si el paciente presenta antecedentes de alcoholismo, cirrosis o cualquier alteración hepática, debe inclinarse el diagnóstico hacia un sangrado alto de origen varicoso; por el contrario, se debe sospechar de un sangrado alto de origen no varicoso cuando el paciente tiene antecedente de consumo de AINES o Ácido Acetilsalicílico (ASA) ${ }^{47,48}$.

Si el origen de la hemorragia se sitúa bajo el ángulo de Treizz, el diagnóstico imagenológico se basa en los hallazgos a la colonoscopia debido a que se estaría frente a un sangrado digestivo inferior ${ }^{49}$.

A continuación se describen algunas de las ayudas diagnósticas y terapéuticas utilizadas en el abordaje de un sangrado digestivo:

\section{Endoscopia superior}

Esta ayuda diagnóstica es considerada como primera opción en pacientes con sangrado digestivo superior, 
permite identificar la causa del sangrado digestivo superior en el $95 \%$ de los casos, además de la causa se debe describir el tipo, localización y cantidad de lesiones, también debe dar constancia de si el sangrado es activo o si posee hallazgos que sugieran una hemorragia reciente ${ }^{44,49}$.

Según la ESGE, la endoscopia debe ser llevada a cabo durante las 24 horas siguientes al episodio hemorrágico en los pacientes que no tienen un diagnóstico confirmado para disminuir el tiempo de estancia hospitalaria, resangrado y evaluar la necesidad de una intervención quirúrgica; también se puede considerar un uso muy temprano de esta ayuda diagnóstica (primeras 12 horas) en pacientes con inestabilidad hemodinámica que persiste a pesar de reanimación con líquidos; otros de sus usos son la evacuación de hematomas y el hallazgo de otras lesiones relacionadas con el cuadro clínico ${ }^{45,49}$. Su sensibilidad alcanza entre el 92 y $98 \%$ mientras que su porcentaje de especificidad está entre el 30 y $100 \% 49$. Este método diagnóstico puede presentar complicaciones para el paciente tales como perforaciones del tubo digestivo, aspiración y aumento del sangrado durante el procedimiento ${ }^{49}$.

La ESGE recomienda la terapia endoscópica, la cual puede realizarse mediante tres modalidades ${ }^{45}$ :

1. Inyección de medicamentos como epinefrina, agentes esclerosantes y fibrina. Se ha evidenciado una disminución en el resangrado y en la cirugía de emergencia cuando se combina epinefrina con otro agente, pero no se ha disminuído la mortalidad comparada con la monoterapia con epinefrina.

2. La terapia térmica por electrocoagulación ha demostrado ser efectiva en alcanzar la hemostasia primaria, reducir la recurrencia del sangrado, la necesidad de cirugía urgente y la mortalidad.

3. La terapia mecánica con clips también ha demostrado reducir el resangrado, pero no se ha evidenciado una mejoría en la hemostasia de los pacientes.

En pacientes con sangrado activo, la Asociación Europea recomienda combinar la inyección de epinefrina con terapia térmica, mecánica o inyección de un agente esclerosante ${ }^{45}$.

\section{Angiografía por tomografía computarizada}

Es utilizada en los casos en que la endoscopia no permite localizar el origen del sangrado; permite visualizar el contraste entre la mucosa intestinal y el contenido hemorrágico cuando el sangrado es mayor a $0,5 \mathrm{ml} / \mathrm{min}^{49}$. Posee una sensibilidad y especificidad del 86 y $95 \%$ respectivamente ${ }^{49}$. Es un método mínimamente invasivo que también permite diagnosticar neoplasias y demostrar tanto la existencia de un sangrado activo como los estigmas de sangrado reciente. Este examen diagnóstico tiene riesgo en pacientes con nefropatías debido al contraste aplicado ${ }^{49}$.

Angiografía por catéter.

Es más usado en sospecha de sangrado inferior que superior, sin embargo, tiene utilidad en ambos; presenta una especificidad alrededor del $100 \%$ y una sensibilidad entre el 42 y $86 \%$, teniendo en cuenta que la pérdida hemorrágica debe ser de 0,5 a $1,5 \mathrm{ml} / \mathrm{min}$ para que este método diagnóstico detecte el sangrado ${ }^{49}$. Un grupo australiano de gastroenterología recomienda realizarlo en pacientes en los que la endoscopia esté contraindicada o arroje resultados no concluyentes y en algunos pacientes hemodinámicamente inestables; por su parte, la ESGE la recomienda en pacientes con resangrado y en los cuales la endoscopia no haya sido efectiva ${ }^{45,49}$.

Su principal ventaja radica en que es una herramienta diagnóstica y terapéutica, pues pueden infundirse medicamentos vasoconstrictores o hacer embolización mediante este. Puede generar algunas complicaciones como hematomas, pseudoaneruismas, y disminución del flujo sanguíneo intestinal, pero estas se presentan en menos del 10\% de los pacientes ${ }^{49}$.

Las siguientes son indicaciones quirúrgicas para los pacientes con HDA según la ESGE45:

1. Falla en el tratamiento endoscópico.

2. Tratamiento endoscópico contraindicado o imposibilidad para realizarlo.

3. Pacientes con resangrado sin control de hemostasia. 
Septiembre - diciembre

\section{Escalas de clasificación}

Existen escalas para clasificar las diferentes características de los pacientes que presentan una hemorragia digestiva alta, entre ellas se encuentran la clasificación de Forrest, el índice de Rockall (Ver Tabla 3), la escala de Glasgow Blatchford (Ver Tabla 4) y el AIMS $65^{\circ}$ (Ver Tabla 5).

\section{Clasificación de Forrest}

Se basa en las características endoscópicas de las úlceras gastrointestinales, tiene como objetivo clasificar la severidad de la hemorragia de acuerdo a los hallazgos de los estudios imagenológicos; es usada como predictor del riesgo de resangrado y mortalidad, estratificando a los pacientes en 6 grupos como se aprecia en la Tabla $2^{50,51}$.

Tabla 2. Clasificación de Forrest

\begin{tabular}{|c|l|}
\hline Clasificación Forrest & \multicolumn{1}{|c|}{ Característica principal } \\
\hline IA & Hemorragia pulsátil. \\
\hline IB & Hemorragia en napa. \\
\hline IIA & Lesión con vaso visible. \\
\hline IIB & Lesión con coágulo. \\
\hline IIC & Mácula plana. \\
\hline III & Fondo limpio. \\
\hline
\end{tabular}

Fuente: Forrest JA, Finlayson ND, Shearman DJ. Endoscopy in gastrointestinal bleeding. Lancet. 1974;304(7877):394-397.

Se estima que el $90 \%$ de los pacientes clasificados en IA presentan recidiva en el sangrado, por su parte, sólo el $5 \%$ de los pacientes clasificados en III la presentan. Su utilidad no está limitada únicamente a la predicción, sino que a partir de la misma clasificación pueden arrojarse alternativas clínicas relevantes para el manejo médico ${ }^{41}$.

\section{Índice de Rockall (RS)}

Propuesto a razón de un estudio que contó con alrededor de 7000 pacientes por T.A Rockall et al. En 1996; presenta dos componentes: uno clínico de donde surge el índice de Rockall pre-endoscópico o inicial, el otro componente se basa en los hallazgos encontrados en la endoscopia originando de esta manera el índice de Rockall completo; es usado como predictor de mortalidad y resangrado ${ }^{52}$. Presenta las variables de edad, shock, comorbilidades y diagnóstico endoscópico, a las cuales se les asigna una clasificación que se obtiene de la suma del puntaje que obtuvo el paciente en cada una. Desde su creación en Inglaterra ha sido bastante usado ${ }^{53}$.

Entre más alto sea el puntaje obtenido, mayor es el riesgo de muerte y resangrado para el paciente, asís:

- Riesgo bajo: Puntuación $\leq 2$.

- Riesgo intermedio: Puntuación $2-5$.

- Riesgo alto: Puntuación > 6 .

Menos del 5\% de los pacientes con una puntuación menor o igual a 2 resangró y su mortalidad fue prácticamente nula, esto se contrasta con las personas que obtuvieron un puntaje mayor a 2 quienes resangraron y murieron en mayor cuantía ${ }^{52}$.

Tabla 3. Índice de Rockall

\begin{tabular}{|c|c|c|c|c|}
\hline \multirow{2}{*}{ Variable } & \multicolumn{4}{|c|}{ Calificación } \\
\hline & 0 & 1 & 2 & 3 \\
\hline Edad & $<60$ & $60-79$ & $>80$ & \\
\hline Shock & Sin Shock & $\begin{array}{l}\text { Pulsaciones }>100 \\
\text { Presión arterial sistólica } \\
>100 \mathrm{mmHg}\end{array}$ & $\begin{array}{l}\text { Presión arterial sistólica } \\
<100 \mathrm{mmHg}\end{array}$ & \\
\hline Comorbilidad & Ninguna mayor & & $\begin{array}{l}\text { Falla cardíaca, enfermedad } \\
\text { coronaria u otra comorbilidad } \\
\text { mayor. }\end{array}$ & $\begin{array}{l}\text { Falla renal, falla hepática o } \\
\text { cáncer metastásico. }\end{array}$ \\
\hline Diagnóstico endoscópico & Mallory-Weiss & Otro diagnóstico. & Malignidad gastrointestinal. & \\
\hline Evidencia de sangrado & Ninguno & & $\begin{array}{l}\text { Sangre visible, coágulo adherente } \\
\text { o vaso pulsátil. }\end{array}$ & \\
\hline
\end{tabular}

Fuente: Rockall TA, Logan RFA, Devlin HB, Northfield TC. Risk assessment after acute upper gastrointestinal haemorrhage. Gut. 1996;38(3):316-21. 


\section{Escala de Glasgow-Blatchford (GBS)}

Desarrollada a inicios del siglo XXI, es usada en pacientes que presentan sangrado digestivo alto para predecir si requieren o no un tratamiento inmediato, para ello se tiene en cuenta una serie de parámetros serológicos y clínicos, en los primeros se encuentran la úrea y la hemoglobina dividida según el sexo mientras en los segundos se incluyen el pulso y la presentación del episodio acompañada de unas posibles comorbilidades, a estas variables se les asigna una puntuación la cual se aprecia en la Tabla $4^{54}$.

El estudio en el que se publicó por primera vez esta clasificación contó con 1748 pacientes, en los cuales se identificaron de manera apropiada (sensibilidad) el $99 \%$ de los pacientes con un sangrado que requería intervención médica y el 32\% de los que no lo necesitaban (especificidad) ${ }^{54}$.

Tabla 4. Escala de Glasgow Blatchford

\begin{tabular}{|c|c|}
\hline Variable. & Puntaje \\
\hline \multicolumn{2}{|c|}{ Úrea (mmol/L) } \\
\hline $6,5-7,9$ & 2 \\
\hline $8-9,9$ & 3 \\
\hline $10-24,9$ & 4 \\
\hline$>25$ & 6 \\
\hline \multicolumn{2}{|c|}{ Hemoglobina $(\mathrm{g} / \mathrm{dL})$ hombres. } \\
\hline $12-12,9$ & 1 \\
\hline $10-11,9$ & 3 \\
\hline$<10$ & 6 \\
\hline \multicolumn{2}{|c|}{ Hemoglobina (g/dL) mujeres. } \\
\hline $10-11,9$ & 1 \\
\hline$<10$ & 6 \\
\hline \multicolumn{2}{|c|}{ Tensión arterial sistólica $(\mathrm{mmHg})$. } \\
\hline $100-109$ & 1 \\
\hline $90-99$ & 2 \\
\hline$<90$ & 3 \\
\hline \multicolumn{2}{|c|}{ Pulso (Lat/min) } \\
\hline$\geq 100$ & 1 \\
\hline \multicolumn{2}{|c|}{ Presentación clínica y comorbilidad } \\
\hline Presentación con melena & 1 \\
\hline Presentación con síncope & 2 \\
\hline Enfermedad hepática & 2 \\
\hline Insuficiencia cardíaca & 2 \\
\hline
\end{tabular}

Fuente: Blatchford O, Murray WR, Blatchford M. A risk score to predict need for treatment for upper-gastrointestinal haemorrhage. Lancet. 2000;356(9238):1318-21.
Los pacientes que obtienen un puntaje mayor a o son clasificados como de alto riesgo. Esta escala, al no poseer un componente endoscópico, presenta una mayor facilidad para ser usada en la primera instancia de la atención ${ }^{53}$.

\section{Clasificación AIMS65}

Esta clasificación fue publicada en 2011 en un estudio de más de 60000 pacientes; es frecuentemente usada como predictor de mortalidad, aunque no es su única finalidad pues puede hacer lo propio con la duración de la estancia hospitalaria y el costo del tratamiento de los pacientes ${ }^{55}$. Su nombre (AIMS65) surge de las iniciales en el idioma inglés de los cinco factores de riesgo que se usan para obtener el puntaje: albúmina, INR (International Normalized Ratio), alteración del estado mental, presión arterial sistólica y edad; cada factor arroja un puntaje que posteriormente se suma para obtener la calificación definitiva, de tal manera que 0 es el menor y 5 el mayor resultado posible ${ }^{55}$.

Tabla 5. Clasificación AIMS65

\begin{tabular}{|c|c|}
\hline Variable & Puntaje \\
\hline Albúmina $<3 \mathrm{~g} / \mathrm{dl}$ & 1 \\
\hline INR $>1,5$ & 1 \\
\hline Alteración del estado mental & 1 \\
\hline Presión sistólica $\leq 90 \mathrm{mmHg}$ & 1 \\
\hline Edad $>65$ & 1 \\
\hline Total & $0-5$ \\
\hline
\end{tabular}

Fuente: Saltzman JR, Tabak YP, Hyett BH, Sun X, Travis AC, Johannes RS. A simple risk score accurately predicts in-hospital mortality, length of stay, and cost in acute upper GI bleeding. Gastrointest Endosc. 2011;74(6):1215-24.

La inclusión de la albúmina en los criterios presenta verdadera importancia debido a que, además de no pertenecer a las demás clasificaciones, sus bajos niveles séricos están directamente relacionados con la mortalidad de pacientes ${ }^{55}$.

\section{Discusión sobre las escalas de evaluación}

Diferentes autores realizan apreciaciones sobre las escalas de evaluación ya planteadas anteriormente. En un estudio español, Martínez et al..$^{50}$ afirman que la clasificación AIMS65 no sufrió un aumento significativo $(1,85 \pm 0,4)$ en comparación con la escala de Glasgow Blatchford $(12,7 \pm 0,7)$ y el índice de 
Rockall $(6,8 \pm 0,5)$ al evaluar el resangrado. En cuanto a la mortalidad no se encontraron diferencias entre las tres en las curvas ROC: AIMS 0.76 , GBS 0.78 , RS $0.78^{56}$. En los resultados arrojados se evidenció que las tres escalas ya mencionadas fueron útiles para prever la necesidad de transfusión sanguínea: AIMS 0.71 , GBS 0.85 , RS 0.73 , siendo la de escala de Glasgow Blatchford la de mejor desempeño ${ }^{56}$. La AIMS65 destaca sobre las otras escalas debido a que presenta una menor complejidad, lo que le permite ser más usada en la práctica clínica diaria57.

Contrastando la capacidad para predecir qué pacientes son de alto y bajo riesgo entre la escala de Glasgow Blatchford y el índice de Rockall, en un estudio realizado en Taiwán, se obtuvo un 100\% (IC95: 98.3-100) de sensibilidad (S), 3.41\% (IC95: 0.759.65) de especificidad (E), valor predictivo positivo (VPP) del 71.67\%(IC95: 65.4-76) y negativo (VPN) del 100\%(IC95: 30.5-100) con un GBS > 0, mientras que las del RS>2 fueron: S: $96.7 \%$ (IC95: 93.4-98.7) E: 11.4\%(IC95: 5.6-19.9), VPP: 72.7\%(IC95: 67.2-77.8) y VPN: 58.8\%(IC95: 33-81.5). El área bajo la curva (AUC) fue de 0.808 (IC95: 0.756-0.86) para GBS y 0.767 (IC95: 0.713-0.821) para RS, lo que indica que la GBS puede ser más útil para estratificar el riesgo que el RS, pues presenta mayor sensibilidad. En cuanto al la predicción de resangrado, el GBS $>0$ presento $\mathrm{S}$ : 100\% (IC95: 91.1-100) y E: 1.1\% (IC95: 0.25-3.3), el RS>2 obtuvo S: $100 \%$ (IC95: 84.4-100) y E: 6.46\% (IC95: 3.8110.15), por lo que, debido a su baja especificidad, no son buenos predictores de resangrado ${ }^{58}$. Lo expuesto anteriormente discrepa de lo publicado por Tuncer et al. en Turquía, quienes sugieren que el RS>2 tuvo un mejor desempeño con S: 96.4\% (IC95: 81.7-99.9), E: 20\% (IC95: 13.4-28.1), VPP: 21.3\% (IC95: 14.5-29.4), VPN: 96.2\% (IC95: 80.4-99.9) que el GBS>0 que tuvo S: $100 \%$ (IC95: 87.7-100), E: 0\% (IC95: 0-2.9) y VPP: 18.3\% (IC95: 12.5-25.4) para predecir la recurrencia de sangrado, en un estudio que contó con 153 pacientes ${ }^{21}$.

En un estudio multicéntrico que contó con 3012 pacientes residentes en Europa, Norteamérica, Asia y Oceanía, la escala más eficiente para predecir la necesidad de intervención asistencial fue la de Glasgow Blatchford <1 (S:98.6\%, E:34.6\%, VPP: 96.6\% y VPN: $56 \%$ ) frente a AIMS65= (S: 81.6\%, E:49.9\%, VPP: $74.7 \%$ y VPN: 59.9 ) y un RS de o (S: $95.6 \%$, E: $23.4 \%$, VPP: $86.5 \%$ y VPN: $50.9 \%$ ); mientras que en la predicción de mortalidad a 30 días, las clasificaciones AIMS65 (S: 65.8\%, E: 76.2\%, VPP: 18\% y VPN: 96.6\%) y RS (S: 74\%, E: 61.7.\%, VPP: 11.1\% y VPN: 97.3\%) tuvieron un mejor rendimiento que las presentadas por GBS
(S: $88.7 \%$, E: 40.2\%, VPP: 9.9 y VPN: $97.9 \%)^{59}$. Otro estudio publicado por Yaka et al. en una población de 254 personas encontró que GBS (AUC 0.809 [IC95: 0.756-0.856]) y la clasificación AIMS65 (AUC 0.809 [IC95: 0.756-0.856]) tuvieron similar capacidad de predicción de mortalidad a 30 días, en este mismo estudio se evidenció que la GBS (AUC 0.904 [IC95: 0.861-0.937]) tuvo un mejor desempeño para prever la necesidad de transfusión sanguínea que AIMS65 (AUC 0.796 [IC95: 0.742-0.844]) ${ }^{60}$. Dichos hallazgos coinciden con una investigación realizada, en Portugal donde también encontraron que la escala que mayor eficiencia tiene para predecir la necesidad de transfusión de glóbulos rojos era la GBS (AUC 0.826 [IC95: 0.773-0.880]), en contraparte, en un estudio con 278 pacientes estadounidenses, la AIMS65 presentó un mejor rendimiento en predecir mortalidad que la GBS y esta última tuvo un mejor desempeño en predecir la necesidad de transfusión sanguínea ${ }^{61,62}$.

En un estudio iraní de 182 pacientes se comparó la escala de Glasgow Blatchford y el índice de Rockall, la GBS presentó un mayor rendimiento para predecir resangrado (AUC 0.722 vs 0.520 ), mientras el RS hizo lo propio con la mortalidad (AUC 0.648 vs 0.582$)^{63}$.

También se han realizado estudios como el publicado por Budimir et al. en Croacia, en el que ninguna de las tres escalas evaluadas (GBS, AIMS65 y RS) tuvieron un buen desempeño para predecir mortalidad y resangrado ${ }^{64}$. Contrario a lo ocurrido en el estudio de $\mathrm{Gu}$ et al., en el que compararon las mismas escalas en pacientes hospitalizados por HDA y las tres presentaron resultados aceptables para predecir mortalidad, siendo la de mejores resultados la AIMS659.

Hay pocas publicaciones que comparen la clasificación de Forrest con otras. En un estudio con 371 pacientes de un centro de atención coreano, los cuales fueron sometidos a dos endoscopias con una semana de diferencia entre una y otra, se obtuvo como resultado que en la primera imagen el $72,2 \%$ de los pacientes fueron clasificados en el grupo de alto riesgo, es decir, presentaron una úlcera entre el tipo IA y el IIC, entre tanto, la segunda imagen arrojó resultados contrarios en los que el $70,6 \%$ de los pacientes presentó una úlcera correspondiente a la clasificación de bajo riesgo, lo que indica que el riesgo de sangrado y mortalidad son mayores en la primera semana donde se altera la mucosa del tracto digestivo ${ }^{65}$. 
El consenso para el manejo de la hemorragia gastrointestinal alta de la Sociedad Británica de Gastroenterología recomienda clasificar a los pacientes a su ingreso al servicio de urgencias según la escala de Glasgow Blatchford, la cual ha demostrado tener el mejor rendimiento en predecir los desenlaces clínicamente importantes como necesidad de transfusión, tratamiento endoscópico, cirugía y muerte, también se usa la AIMS65 y el Índice de Rockall para predecir mortalidad ${ }^{66}$. Históricamente se ha usado la GBS de o para clasificar a los pacientes de muy bajo riesgo y determinar si se benefician de manejo extrahospitalario, pero actualmente es controversial si ese punto de corte debe ser igual o menor a 1 , incluso el Grupo de Consenso Internacional para el manejo de hemorragia digestiva alta no variceal recomienda tomar el 1 como punto de corte en el GBS, pero no recomiendan usar otra escala como RS o AIMS65 para clasificar los pacientes en la categoría de muy bajo riesgo. Este grupo de expertos también recomienda usar la GBS, RS y AIMS65 de manera individual en todos los pacientes con hemorragia digestiva alta ${ }^{66,67}$.

\section{Conclusiones}

La HDA es la principal emergencia gastroenterológica y continúa siendo una patología de distribución e importancia global por su prevalencia, mortalidad y amplios gastos en salud que debe intervenirse. Su etiología es variable, siendo más frecuentes las úlceras pépticas en las causas no varicosas y las várices esofágicas en las varicosas. El manejo de estos pacientes se basa en un abordaje dirigido a la estabilización y reanimación inicial y diagnóstico temprano en el cual se emplean técnicas endoscópicas, angiográficas y quirúrgicas que pueden usarse también como tratamiento según el tipo de paciente al que se enfrente el clínico, aunque esto depende de los recursos con los que cuente el personal de salud al momento de la atención. Las escalas de evaluación o predicción son recomendadas por diferentes grupos de expertos y guías de práctica clínica alrededor del mundo en la evaluación rutinaria de los pacientes con HDA, siendo la Escala de Glasgow Blatchford la que cuenta con mayores recomendaciones iniciales, sin dejar de lado el Índice de Rockal y la clasificación AIMS65, sin embargo, las diferentes investigaciones realizadas alrededor del mundo plantean diferencias entre los resultados de los estudios que evalúan las actuales escalas de evaluación, lo que sugiere una nueva posibilidad de investigación orientada hacia una eficaz estratificación de los pacientes, buscando que la predicción sea un pilar fundamental en el manejo de las personas que presenten una HDA e impactar de esta manera en los desenlaces de los pacientes.

\section{Conflicto de intereses}

Los autores declaran no tener conflicto de intereses.

\section{Referencias bibliográficas}

1. Armenteros MC, Palomino AB, Mora S, Valladares D, Blanco M. Utilidad del índice de Rockall en pacientes con episodios de hemorragia digestiva alta no variceal. Rev Cub Med Mil. 2014;43(2):176-84.

2. Lu M, Sun G, Huang H, Zhang X, Xu Y, Chen S, et al. Comparison of the Glasgow-Blatchford and Rockall Scores for prediction of nonvariceal upper gastrointestinal bleeding outcomes in Chinese patients. Medicine (Baltimore). 2019;98(21):e15716. PMID: 31124950

3. Lanas A, Dumonceau J-M, Hunt RH, Fujishiro M, Scheiman JM, Gralnek IM, et al. Non-variceal upper gastrointestinal bleeding. Nat Rev Dis Primers. 2018;4:18020. PMID: 29671413

4. Kamboj AK, Hoversten P, Leggett CL. Upper Gastrointestinal Bleeding: Etiologies and Management. Mayo Clin Proc. 2019;94(4):697-703.

5. Stanley AJ. Update on risk scoring systems for patients with upper gastrointestinal haemorrhage. World J Gastroenterol. 2012;18(22):2739-44.

6. Chimbaco DF, Leal MA, González JP, Caviedes G. Factores relacionados a hemorragia gastrointestinal alta en pacientes de la unidad de cuidados intensivos pese a la profilaxis. Rev Méd Risaralda. 2014;20(1):9-13.

7. Lip HT, Heah HT, Huei TJ, Premaa S, Sarojah A. Rockall risk score in predicting 30 days non-variceal upper gastrointestinal rebleeding in a Malaysian population. Med J Malaysia. 2016;71(5):225-30.

8. Cassana A, Scialom S, Segura ER, Chacaltana A. Estudio de validación diagnóstica de la escala de Glasgow-Blatchford para la predicción de mortalidad en pacientes con hemorragia digestiva alta en un hospital de Lima, Perú (junio 2012-diciembre 2013). Rev Esp Enferm Dig. 2015;107(8):476-82.

9. Gu L, Xu F, Yuan J. Comparison of AIMS65, Glasgow-Blatchford and Rockall scoring approaches in predicting the risk of inhospital death among emergency hospitalized patients with upper gastrointestinal bleeding: a retrospective observational study in Nanjing, China. BMC Gastroenterol. 2018;18(1):98.

10. Contreras-Omaña R, Alfaro-Reynoso JA, Cruz-Chávez CE, Velarde-Ruiz A, Flores-Ramírez DI, Romero-Hernández I, et al. The Progetto Nazionale Emorragia Digestiva (PNED) system vs. the Rockall score as mortality predictors in patients with nonvariceal upper gastrointestinal bleeding: A multicenter prospective study. Rev Gastroenterol Mex. 2017;82(2):123-8.

11. Melgar LM, Botello MV, Melgar C, Santofimio D, Isaías H. Causas más frecuentes de Hemorragia en Tubo Digestivo Alto de origen no variceal en paciente mayor de 50 años en Colombia. RNM. 2018;4(2):14-21.

12. Páez O. Guía práctica clínica. Hemorragia digestiva aguda. Salud Uninorte. 2006;22(2):195-206.

13. Bozkurt MA, Peker KD, Unsal MG, Yırgın H, Kahraman İ, Alış H. The Importance of Rockall Scoring System for Upper Gastrointestinal Bleeding in Long-Term Follow-Up. Indian J Surg. 2017;79(3):188-91.

14. Iino C, Shimoyama T, Igarashi T, Aihara T, Ishii K, Sakamoto J, et al. Validity of the Pre-endoscopic Scoring Systems for the Prediction of the Failure of Endoscopic Hemostasis in Bleeding Gastroduodenal Peptic Ulcers. Intern Med. 2018;57(10):1355-60.

15. Park SW, Song YW, Tak DH, Ahn BM, Kang SH, Moon HS, et al. 
The AIMS65 Score Is a Useful Predictor of Mortality in Patients with Nonvariceal Upper Gastrointestinal Bleeding: Urgent Endoscopy in Patients with High AIMS65 Scores. Clin Endosc. 2015; 48(6):522-527.

16. Dong Z, Wang J, Zhan T, Zhang H, Yi L, Xu S. A New Scoring System to Predict Poor Clinical Outcomes in Acute Nonvariceal Upper Gastrointestinal Bleeding Patients with High-Risk Stigmata. Gastroenterol Res Pract. 2018; 2018:5032657.

17. Espinoza-Rios J, Aguilar V, Bravo EA, Pinto J, Huerta-Mercado J. Comparación de los scores Glasgow-Biatchford, Rockall y AIMS65 en pacientes con hemorragia digestiva alta en un hospital de Lima, Peru. Rev gastroenterol Perú. 2016;36(2):143-152.

18. Solaz LO, Jordán AD, Moya OA, Concepcción OA, Méndez DC. Comportamiento de la hemorragia digestiva alta en el quinquenio 2009 a 2013. Rev. Med. Electrón. 2017; 39(3):432-442.

19. Meltzer AC, Klein JC. Upper gastrointestinal bleeding: patient presentation, risk stratification, and early management. Gastroenterol Clin North Am. 2014;43(4):665-675.

20. Chi TY, Zhu HM, Zhang M. Risk factors associated with nonsteroidal anti-inflammatory drugs (NSAIDs)-induced gastrointestinal bleeding resulting on people over 60 years old in Beijing. Medicine (Baltimore). 2018;97(18):e0665.

21. Tuncer H, Yardan T, Akdemir HU, Ayyildiz T. Comparison of four scoring systems for risk stratification of upper gastrointestinal bleeding. Pak J Med Sci. 2018;34(3):649-54.

22. Luo PJ, Lin XH, Lin CC, Luo JC, Hu HY, Ting PH, et al. Risk factors for upper gastrointestinal bleeding among aspirin users: An old issue with new findings from a population-based cohort study. J Formos Med Assoc. 2019;118(5):939-944.

23. Lee DH, Lee KM, Lee SM, Lee BK, Cho YS, Choi G, et al. Performance of Three Scoring Systems in Predicting Massive Transfusion in Patients with Unstable Upper Gastrointestinal Hemorrhage. Yonsei Med J. 2019;60(4):368-374.

24. Shih PC, Liu SJ, Li ST, Chiu AC, Wang PC, Liu LY. Weekend effect in upper gastrointestinal bleeding: a systematic review and metaanalysis. PeerJ. 2018 Jan 12;6:e4248. PubMed PMID: 29340247.

25. Jafar W, Jafar AJN, Sharma A. Upper gastrointestinal haemorrhage: an update. Frontline Gastroenterol. 2016;7(1):32-40.

26. Crooks CJ, West J, Card TR. Upper gastrointestinal haemorrhage and deprivation: a nationwide cohort study of health inequality in hospital admissions. Gut. 2012;61:514-20.

27. Elsebaey MA, Elashry H, Elbedewy TA, Elhadidy AA, Esheba NE, Ezat S, et al. Predictors of in-hospital mortality in a cohort of elderly Egyptian patients with acute upper gastrointestinal bleeding. Medicine (Baltimore). 2018 Apr;97(16):e0403. PubMed PMID: 29668596.

28. Choe JW, Kim SY, Hyun JJ, Jung SW, Jung YK, Koo JS, Yim HJ, Lee SW. Is the AIMS 65 Score Useful in Prepdicting Clinical Outcomes in Korean Patients with Variceal and Nonvariceal Upper Gastrointestinal Bleeding? Gut Liver. 2017;11(6):813-20.

29. Ko IG, Kim SE, Chang BS, Kwak MS, Yoon JY, Cha JM, et al. Evaluation of scoring systems without endoscopic findings for predicting outcomes in patients with upper gastrointestinal bleeding. BMC Gastroenterol. 2017;17(1):159-66.

30. Lanas A, Chan FKL. Peptic ulcer disease. Lancet. 2017;390(10094):613-24.

31. Stewart DJ, Ackroyd R. Peptic ulcers and their complications. Surgery. 2011;29(11):568-74

32. Søreide K, Thorsen K, Harrison EM, Bingener J, Møller $\mathrm{MH}$, Ohene-Yeboah M, et al. Perforated peptic ulcer. Lancet. 2015;386(10000):1288-98.

33. Kempenich JW, Sirinek KR. Acid Peptic Disease. Surg Clin North Am. 2018;98(5): 933-44.

34. Kavitt RT, Lipowska AM, Anyane-Yeboa A, Gralnek IM. Diagnosis and Treatment of Peptic Ulcer Disease. Am J Med. 2019;132(4):447-56.

35. Lo GH. Management of acute esophageal variceal hemorrhage. Kaohsiung J Med Sci. 2010;26(2):55-67.

36. Pandey V, Patil M, Patel R, Chaubal A, Ingle M, Shukla A. Prevalence of splenic vein thrombosis and risk of gastrointestinal bleeding in chronic pancreatitis patients attending a tertiary hospital in western India. J Family Med Prim Care. 2019;8(3):818-22.

37. Shao XD, Qi XS, Guo XZ. Esophageal Stent for Refractory Variceal Bleeding: A Systemic Review and Meta-Analysis. Biomed Res Int. 2016;2016:4054513. Disponible en: https://www.hindawi. com/journals/bmri/2016/4054513/
38. Narváez RM, Cortez CA, González JA, Tamayo JL, Zamarripa F, Torre A, et al. Consenso Mexicano de Hipertensión Portal. Rev Gastroenterol Mex. 2013;78(2):92-113.

39. D'Amico G, Garcia-Tsao G, Pagliaro L. Natural history and prognostic indicators of survival in cirrhosis: a systematic review of 118 studies. J Hepatol. 2006;44(1):217-31.

40. Cybułka B. Malory-Weis syndrome based on own experience diagnostics and modern principles of management. Pol Przegl Chir. 2016;88(2):77-86.

41. Lee S, Ahn JY, Jung HY, Jung KW, Lee JH, Kim DH, et al. Effective endoscopic treatment of Mallory-Weiss syndrome using Glasgow-Blatchford score and Forrest classification. J Dig Dis. 2016;17(10):676-84.

42. Rich K. Overview of Mallory-Weiss syndrome. J Vasc Nurs. 2018;36(2):91-3. Disponible en: https://www.sciencedirect.com/ journal/journal-of-vascular-nursing/vol/36/issue/2

43. Feinman M, Haut ER. Upper gastrointestinal bleeding. Surg Clin North Am. 2014;94(1):43-53. Disponible en: https://www. surgical.theclinics.com/article/S0039-6109(13)00153-9/abstract

44. Betés M, Muñoz-Navas M. Hemorragia digestiva alta no varicosa. Medicine. 2016;12(2):82-91.

45. Gralnek IM, Dumonceau JM, Kuipers EJ, Lanas A, Sanders DS, Kurien M, et al. Diagnosis and management of nonvariceal upper gastrointestinal hemorrhage: European Society of Gastrointestinal Endoscopy (ESGE) Guideline. Endoscopy. 2015;47(10):1-46. Disponible en: https://eref.thieme.de/ ejournals/1438-8812_2015_10\#/10.1055-s-0034-1393172

46. Saleem S, Thomas AL. Management of Upper Gastrointestinal Bleeding by an Internist. Cureus. 2018;10(6):e2878. Disponible en: https://www.cureus.com/articles/12613-management-ofupper-gastrointestinal-bleeding-by-an-internist

47. Lu Y, Loffroy R, Lau JY, Barkun A. Multidisciplinary management strategies for acute non-variceal upper gastrointestinal bleeding. Br J Surg. 2014;101(1):e34-50. Disponible en: https://bjssjournals. onlinelibrary.wiley.com/doi/full/10.1002/bjs.9351

48. Biecker E. Diagnosis and therapy of non-variceal upper gastrointestinal bleeding. World J Gastrointest Pharmacol Ther. 2015;6(4):172-82. Disponible en: https://www.wjgnet.com/21505349/full/v6/i4/172.htm

49. Kim BS, Li BT, Engel A, Samra JS, Clarke S, Norton ID, et al. Diagnosis of gastrointestinal bleeding: A practical guide for clinicians. World J Gastrointest Pathophysiol. 2014;5(4):46778. Disponible en: https://www.wjgnet.com/2150-5330/full/v5/ i4/467.htm

50. Giese A, Grunwald C, Zieren J, Büchner NJ, Henning BF. Use of the Complete Rockall Score and the Forrest Classification to Assess Outcome in Patients with Non-variceal Upper Gastrointestinal Bleeding Subject to After-hours Endoscopy: A Retrospective Cohort Study. West Indian Med J. 2014;63(1):2933. Disponible en: https://pubmed.ncbi.nlm.nih.gov/25303191/

51. Forrest JA, Finlayson ND, Shearman DJ. Endoscopy in gastrointestinal bleeding. Lancet. 1974;304(7877):394-397. Disponible en: https://www.sciencedirect.com/science/article/ abs/pii/S014067367491770X

52. Rockall TA, Logan RFA, Devlin HB, Northfield TC. Risk assessment after acute upper gastrointestinal haemorrhage. Gut. 1996;38(3):316-21.

53. Winograd R, Infante M, Guisado Y, Angulo O, González I, William E. Escalas de predicción en el pronóstico del paciente con hemorragia digestiva alta no varicosa. Rev Cuba Med Mil. 2015;44(1):73-85

54. Blatchford O, Murray WR, Blatchford M. A risk score to predict need for treatment for upper-gastrointestinal haemorrhage. Lancet. 2000;356(9238):1318-21.

55. Saltzman JR, Tabak YP, Hyett BH, Sun X, Travis AC, Johannes RS. A simple risk score accurately predicts in-hospital mortality, length of stay, and cost in acute upper GI bleeding. Gastrointest Endosc. 2011;74(6):1215-24. Disponible en: https:/www. sciencedirect.com/science/article/abs/pii/S0016510711018633

56. Martínez-Cara JG, Jiménez-Rosales R, Úbeda-Muñoz M, de Hierro ML, de Teresa J, Redondo-Cerezo E. Comparison of AIMS65, Glasgow-Blatchford score, and Rockall score in a European series of patients with upper gastrointestinal bleeding: performance when predicting in-hospital and delayed mortality. United European Gastroenterol J. 2016;4(3):371-79. Disponible en: https://journals.sagepub.com/doi/10.1177/2050640615604779 
57. Jung SH, Oh JH, Lee HY, Jeong JW, Go SE, You CR, Jeon EJ, Choi SW. Is the AIMS65 score useful in predicting outcomes in peptic ulcer bleeding? World J Gastroenterol. 2014;20(7):184651. Disponible en: https://www.wjgnet.com/1007-9327/full/v20/ i7/1846.htm

58. Wang CH, Chen YW, Young YR, Yang CJ, Chen IC. A prospective comparison of 3 scoring systems in upper gastrointestinal bleeding. Am J Emerg Med. 2013;31(5):775-78. Disponible en https://www.ajemjournal.com/article/S0735-6757(13)00024-7/ fulltext

59. Stanley AJ, Laine L, Dalton HR, Ngu JH, Schultz M, Abazi R, et al. Comparison of risk scoring systems for patients presenting with upper gastrointestinal bleeding: international multicentre prospective study. BMJ. 2017; 356.

60. Yaka E, Yılmaz S, Doğan NÖ, Pekdemir M. Comparison of the Glasgow-Blatchford and AIMS65 scoring systems for risk stratification in upper gastrointestinal bleeding in the emergency department. Acad Emerg Med. 2015;22(1):22-30. Disponible en: https://pubmed.ncbi.nlm.nih.gov/25556538/

61. Cúrdia Gonçalves T, Barbosa M, Xavier S, Boal Carvalho P, Firmino Machado J, Magalhães J, et al. Optimizing the Risk Assessment in Upper Gastrointestinal Bleeding: Comparison of 5 Scores Predicting 7 Outcomes. GE Port J Gastroenterol. 2018;25(6):299-307.

62. Hyett BH, Abougergi MS, Charpentier JP, Kumar NL, Brozovic
S, Claggett BL, et al. The AIMS65 score compared with the Glasgow-Blatchford score in predicting outcomes in upper GI bleeding. Gastrointest Endosc. 2013;77(4):551-57.

63. Mokhtare M, Bozorgi V, Agah S, Nikkhah M, Faghihi A, Boghratian A, et al. Comparison of Glasgow-Blatchford score and full Rockall score systems to predict clinical outcomes in patients with upper gastrointestinal bleeding. Clin Exp Gastroenterol. 2016;9:337-43.

64. Budimir I, Gradišer M, Nikolić M, Baršić N, Ljubičić N, Kralj D, et al. Glasgow Blatchford, pre-endoscopic Rockall and AIMS65 scores show no difference in predicting rebleeding rate and mortality in variceal bleeding. Scand. J. Gastroenterol. 2016;51(11):1375-79.

65. Lee JY, Kim CG, Cho SJ, Kim YI, Choi IJ. Is the Reinitiation of Antiplatelet Agents Safe at 1 Week after Gastric Endoscopic Submucosal Dissection? Assessment of Bleeding Risk Using the Forrest Classification. Gut Liver. 2017;11(4):489-96

66. Siau K, Hearnshaw S, Stanley AJ, Estcourt L, Rasheed A, Walden A, et al. British Society of Gastroenterology (BSG)led multisociety consensus care bundle for the early clinical management of acute upper gastrointestinal bleeding. Frontline Gastroenterol. 2020;11(4):311-23.

67. Barkun AN, Almadi M, Kuipers EJ, Laine L, Sung J, Tse F, et al. Management of Nonvariceal Upper Gastrointestinal Bleeding: Guideline Recommendations From the International Consensus Group. Ann Intern Med. 2019;171(11):805-22. 